\title{
Chemokine RANTES and IL-1 $\beta$ in mild therapeutic hypothermia-treated patients after out-of-hospital sudden cardiac arrest
}

\author{
Jakub Podolec ${ }^{1}$, Aleksander Trąbka-Zawicki ${ }^{1}$, Rafał Badacz $^{1}$, Mateusz Siedliński ${ }^{2}$, Marek Tomala ${ }^{1}$, \\ Krzysztof Bartuś ${ }^{3}$, Jacek Legutko ${ }^{1}$, Tadeusz Przewłocki ${ }^{1}$, Krzysztof Żmudka ${ }^{1}$, Anna Kabłak-Ziembicka ${ }^{1}$ \\ 1Department of Interventional Cardiology, Jagiellonian University College of Medicine, John Paul II Hospital, Krakow, Poland \\ ${ }^{2}$ Department of Internal and Agricultural Medicine, Faculty of Medicine, Jagiellonian University Medical College, Krakow, Poland \\ ${ }^{3}$ Department of Cardiovascular Surgery and Transplantology, Jagiellonian University College of Medicine, John Paul II Hospital, Krakow, Poland
}

Adv Interv Cardiol 2019; 15, 1 (55): 98-106 DOI: https://doi.org/10.5114/aic.2019.83653

\begin{abstract}
A bstract
Introduction: CCL5/RANTES and IL-1 1 , which regulate the immune response, may have an impact on survival in patients with acute coronary syndrome (ACS) and sudden cardiac arrest (SCA).

Aim: To evaluate levels of CCL5/RANTES and IL-1 in patients with ACS complicated by SCA, treated with coronary angioplasty (PCI) and mild therapeutic hypothermia (MTH), and these chemokines' impact on the 30- and 180-day survival.

Material and methods: Thirty-three unconscious patients admitted after SCA with ACS underwent PCI and MTH treatment. CCL5/RANTES and IL-1 $\beta$ were evaluated on admission (T0), at 12-24 h (T1) and at 48-72 h (T2). All-cause mortality was recorded at 30 and 180 days.

Results: We observed a statistically significant decrease in median levels of CCL/RANTES at T0, T1 and T2 (24.69 ng/ml vs. $3.89 \mathrm{ng} / \mathrm{ml}$ vs. $2.71 \mathrm{ng} / \mathrm{ml} ; p<0.001)$, and significant differences in median levels of IL-1 $(0.196 \mathrm{pg} / \mathrm{ml} \mathrm{vs.} 0.171 \mathrm{pg} / \mathrm{ml} \mathrm{vs} .0 .214 \mathrm{pg} / \mathrm{ml}$; $p=0.034)$. Initial levels of CCL5/RANTES and IL-1 $\beta$ correlated significantly $(r=-0.360 ; p=0.045)$. At T2, CCL5/RANTES correlated with the maximum levels of hs-TnT and CK-MB $(r=-0.594 ; p<0.001$ and $r=-0.389 ; p=0.030)$, and at T0 with BNP $(r=-0.521$; $p=0.003)$. Mortality rate at 30 days and 180 days was $18.2 \%$ and $45.5 \%$, respectively. At 30 days, we observed a trend to significance for IL-1 $\beta$ at T0 and T1 ( $p=0.078$ and $p=0.079)$, but not for CCL5/RANTES ( $p=0.284$ and $p=0.351)$. For 180-day survival curves, only the IL-1 $\beta$ level at T1 was associated with mortality $(p=0.028)$.

Conclusions: Although CCL5/RANTES levels correlate with cardiac injury and heart failure markers and they decrease during MTH, they failed to predict early and late mortality. In contrast, IL-1 $\beta$ level was associated with 180-day survival.
\end{abstract}

Key words: chemokine RANTES, IL-1 1 , mild therapeutic hypothermia, sudden cardiac arrest.

S u m m a ry

Chemokine CCL5/RANTES is present in early stages of the inflammatory process in patients with out-of-hospital cardiac arrest, which then significantly decreases during mild therapeutic hypothermia. The MTH leads to a decrease in the inflammatory response measured by IL-1 $\beta$. The level of IL-1 $\beta$ measured during MTH was significantly associated with long-term all-cause mortality. CCL5/RANTES levels correlate with myocardial injury and BNP levels.

\section{Introduction}

The most common cause of sudden cardiac arrest (SCA) is acute coronary syndrome (ACS). In Poland, over 177000 patients died due to cardiac diseases in 2013, which accounted for $45.8 \%$ of all deaths [1]. Out-of-hos- pital cardiac arrest (OHCA) is associated with a particularly poor prognosis and low survival rates. Moreover, these patients are at risk for neurological damage [2, 3]. Post-resuscitation syndrome includes complex damage of individual organs caused by ischemia-reperfusion in-

\section{Corresponding author:}

Jakub Podolec MD, PhD, Department of Interventional Cardiology, Jagiellonian University Medical College, John Paul II Hospital, 80 Pradnicka St, 31-202 Krakow, Poland, e-mail: jjpodolec@gmail.com

Received: 12.02.2019, accepted: 26.02.2019. 
jury [4]. To protect against organ damage, mild therapeutic hypothermia (MTH), which is defined as a controlled decrease in body temperature to $32-34^{\circ} \mathrm{C}[5,6]$, was recently introduced [7, 8].

An Australian study by Bernard et al. and a European study by the Hypothermia after Cardiac Arrest (HACA) working group have documented that the use of therapeutic hypothermia was associated with clinically significant neurological improvement and a 14\% improvement in survival 6 months after resuscitation compared to the control group without hypothermia induction (59\% vs. $45 \%, p<0.02)[5,6]$. The results of these studies have suggested that current guidelines of the European Resuscitation Council (ERC) and the European Society of Cardiology (ESC) recommend therapeutic hypothermia and/or (recently) target temperature management (TTM) in patients with OHCA, regardless of the rhythm which initiated sudden cardiac arrest [9].

However, it is controversial whether MTH might trigger infectious complications through a pro-inflammatory effect (including sepsis) or by creating a "sepsis-like" syndrome via an increase in pro-inflammatory cytokines, including interleukin (IL)-1 $\beta, \mathrm{IL}-8$, and tumor necrosis factor (TNF)- $\alpha$ [10]. A significant correlation has been demonstrated between chemokine (C-C motif) ligand 5 (CCL5), also known as regulated on activation, normal $\mathrm{T}$ cell expressed and secreted (RANTES), IL-1 $\beta$ level, and mortality risk in patients with acute coronary syndromes [11-13]. Chemokine CCL5/RANTES level is a marker of coronary artery disease severity $[14,15]$. An expanded panel of inflammatory parameters (CCL5/RANTES and IL-1 $\beta$ level) in association with basic measurements (C-reactive protein (CRP), leukocytes, neutrophils) during therapeutic hypothermia may have potential benefits, including prevention of undesirable effects, for optimization of anti-inflammatory therapy or predicting prognosis in patients with ACS complicated by SCA undergoing percutaneous coronary interventions.

\section{Aim}

The aim of the study was to evaluate the activity of chemokines which regulate immune cell vascular chemotaxis, including CCL5/RANTES and IL-1 $\beta$, and their potential impact on 30-day and 180-day prognosis in patients with ACS complicated by SCA treated with coronary angioplasty and MTH.

\section{Material and methods}

The study included 33 unconscious patients ( 27 male, mean age: $66.1 \pm 10.8$ years) admitted after out-of-hospital cardiac arrest as a result of ACS between July 2011 and June 2016. Inclusion criteria were as follows: ACS complicated by OHCA with return of spontaneous circulation (ROSC) and treated using MTH. Patients needed to be $>18$ years of age and underwent percutaneous coronary intervention $(\mathrm{PCl})$ of a culprit artery with implantation of a coronary stent. Patients were excluded from MTH if they had a known coagulopathy or active bleeding. All patients had MTH induction in accordance with European Society of Cardiology guidelines [16]. Written informed consent was obtained from patients after regaining consciousness following cardiac arrest. The Jagiellonian University Ethics Committee in Krakow approved the protocol.

\section{Cardiac revascularization and patient management}

All patients underwent early $\mathrm{PCl}$ and received optimal medical treatment according to current guidelines [17]. Each patient received periprocedural unfractionated heparin intravenously according to weight and dual antiplatelet therapy via a nasogastric tube. During the procedure, GP IIb/IIla was administered at the discretion of the interventional cardiologist. A drug-eluting stent was implanted in $26(78.8 \%)$ patients, while a bare metal stent was implanted in 4 (12.1) patients. Balloon angioplasty without stent implantation was performed in 2 (6.1\%) patients, while in 1 patient, balloon introduction was unsuccessful. SYNTAX score, as well as TIMI scales (pre- and post-PCI), were assessed in all subjects.

\section{Inflammatory biomarkers}

Blood samples were drawn from an antecubital vein on admission ( $\left.T_{0}\right)$, at 12-24 $\mathrm{h}\left(\mathrm{T}_{1}\right)$, and 48-72 $\mathrm{h}\left(\mathrm{T}_{2}\right)$ after $\mathrm{PCl}$ and placed in a collection tube. Plasma was centrifuged for $15 \mathrm{~min}$ at $1600 \times \mathrm{g}$ at $4^{\circ} \mathrm{C}$. Collected serum aliquots were immediately stored at $\leq-70^{\circ} \mathrm{C}$ for further analysis of CCL5/RANTES and IL-1 $\beta$. Biomarker serum levels were determined by ELISA (Human CCL5/RANTES Immunoassay no. DRNO0B, Human IL1 $\beta 1$ Immunoassay R\&D systems, Minneapolis, MN, USA) following the manufacturer's instructions.

\section{Blood sampling}

Baseline blood samples, including biochemical tests, CRP, BNP, coagulation, blood gas analysis, and myocardial injury markers were taken on admission to the hospital $\left(T_{0}\right)$, and then at $T_{1}(12-24 \mathrm{~h})$ and $T_{2}(48-72 \mathrm{~h})$. Average core temperatures were as follows: at $\mathrm{T}_{0} 35.4 \pm 0.6^{\circ} \mathrm{C}$; at $\mathrm{T}_{1} 32.4 \pm 0.5^{\circ} \mathrm{C}$; and at $\mathrm{T}_{2} 36.6 \pm 0.4^{\circ} \mathrm{C}$.

\section{Mild therapeutic hypothermia protocol}

To induce hypothermia, infusion of cold saline $(\mathrm{NaCl}$ $0.9 \%$ at $4^{\circ} \mathrm{C}$ ) was initiated during $\mathrm{PCl}$. After $\mathrm{PCl}$ and transportation to the intensive care unit, therapeutic hypothermia was achieved through the use of an endovascular cooling device (Coolgard, Zoll Medical Corporation Chelmsford, MA, USA). According to the protocol, cooling was maintained for $24 \mathrm{~h}$ with a target temperature of $33^{\circ} \mathrm{C}$. After reaching the target temperature, rewarming 
was performed at a rate of $0.2^{\circ} \mathrm{C} / \mathrm{h}$. All patients during $\mathrm{MTH}$ received intravenous injection of an analgesic opioid and a sedation drug. Additionally, neuromuscular blockade (NMB) was often used in accordance with the protocol of MTH.

\section{Patient discharge and follow-up}

The primary endpoint was to establish the pattern of CCL5/RANTES and IL- $1 \beta$ changes at 3 time points, and to determine correlations between CCL5/RANTES and IL-1 $\beta$ along with inflammatory, coagulation, and myocardial injury/heart failure biomarkers.

The secondary endpoint was to analyze the relationship between CCL5/RANTES, IL-1 $\beta$, and the 30-day and 180-day mortality. Neurological outcome was assessed at discharge according to the Pittsburgh Cerebral Perfor- mance Category (CPC) $[18,19]$. CPC scores 1 and 2 were defined as favorable neurological outcomes, whereas CPC scores 3, 4, and 5 were associated with a poor neurological outcome.

\section{Statistical analysis}

The data are presented as mean with standard deviations or medians with interquartile range. Continuous variables were compared with Student's t-test for normally distributed variables or with the non-parametric Mann-Whitney $U$ test for non-normally distributed variables. The $\chi^{2}$ test was used to compare proportions of categorical variables. Means of the analyzed parameters across groups were tested by analysis of variance (ANOVA).

Correlations were assessed by the Pearson correlation test or by Spearman's rank test. Survival analysis

Table I. Characteristics of patients undergoing mild therapeutic hypothermia (MTH)

\begin{tabular}{|c|c|}
\hline Variables & $\mathrm{MTH}^{*}(n=33)$ \\
\hline Age [years] & $66.1 \pm 10.8$ \\
\hline Male sex, $n(\%)$ & 27 (81.8\%) \\
\hline Systolic blood pressure [mm Hg] & $120(102-147)$ \\
\hline Diastolic blood pressure [mm Hg] & $80(65-91)$ \\
\hline History of hypertension & $25(75.8 \%)$ \\
\hline Diabetes mellitus & $11(33.3 \%)$ \\
\hline Hypercholesterolemia & $20(60.6 \%)$ \\
\hline Obesity & $13(39.4 \%)$ \\
\hline Active smoker & $10(30.3 \%)$ \\
\hline Prior myocardial infarction & $8(24.2 \%)$ \\
\hline Prior PCl & $2(6.1 \%)$ \\
\hline Prior CABG & $2(6.1 \%)$ \\
\hline Prior stroke & $5(15.2 \%)$ \\
\hline \multicolumn{2}{|l|}{ Circumstances of sudden cardiac arrest: } \\
\hline Ventricular tachycardia/ventricular fibrillation & $28(84.8 \%)$ \\
\hline Asystole & $3(9.1 \%)$ \\
\hline Pulseless electrical activity & $2(6.1 \%)$ \\
\hline Basic life support/advanced life support & $23(69.7 \%)$ \\
\hline Time to return of spontaneous circulation [min] & $23 \pm 16$ \\
\hline Preserved pupillary reflex & $23(69.7 \%)$ \\
\hline Glasgow Coma Scale $\leq 4$ & $21(63.6 \%)$ \\
\hline Glasgow Coma Scale > 4 & $12(36.4 \%)$ \\
\hline \multicolumn{2}{|l|}{ Clinical state at admission to hospital } \\
\hline Cardiogenic shock at admission & $8(24.2 \%)$ \\
\hline STEMI & $20(60.6 \%)$ \\
\hline \multicolumn{2}{|l|}{ Course of hospitalization: } \\
\hline Time from cardiac arrest to $\mathrm{PCI}$ [min] & $122.1(60.1)$ \\
\hline Acute coronary occlusion & $16(48.5 \%)$ \\
\hline \multicolumn{2}{|l|}{ Extent of coronary artery disease: } \\
\hline 1 -vessel & $14(42.4 \%)$ \\
\hline 2-vessel & $10(30.3 \%)$ \\
\hline 3-vessel & $9(27.3 \%)$ \\
\hline
\end{tabular}

\begin{tabular}{|c|c|}
\hline Variables & MTH* $^{*}(n=33)$ \\
\hline Left main stenosis & $1(3.0 \%)$ \\
\hline SYNTAX score & $20(6.5-29.5)$ \\
\hline Surgical revascularization & 0 \\
\hline Bare-metal stents used & $4(12.1 \%)$ \\
\hline Drug-eluting stents used & $26(78.8 \%)$ \\
\hline Only balloon angioplasty & $2(6.1 \%)$ \\
\hline Failed PCl & $1(3.0 \%)$ \\
\hline \multicolumn{2}{|l|}{ TIMI flow pre-PCI: } \\
\hline 0 & $18(54.5 \%)$ \\
\hline 1 & $1(3.0 \%)$ \\
\hline 2 & $2(6.0 \%)$ \\
\hline 3 & $12(36.4 \%)$ \\
\hline \multicolumn{2}{|l|}{ TIMI flow post-PCI: } \\
\hline 0 & $3(9.1 \%)$ \\
\hline 1 & $1(3.0 \%)$ \\
\hline 2 & $0(0.0 \%)$ \\
\hline 3 & $29(87.9 \%)$ \\
\hline \multicolumn{2}{|l|}{ Thienopyridine use: } \\
\hline Clopidogrel & $12(36.4 \%)$ \\
\hline Ticagrelor & $21(63.6 \%)$ \\
\hline Cardiogenic shock (after admission) & $17(51.5 \%)$ \\
\hline Intra-aortic balloon counterpulsation 0 & $3(9.1 \%)$ \\
\hline Left ventricular ejection fraction max. & $44(14.8 \%)$ \\
\hline Pneumonia & $24(72.7 \%)$ \\
\hline Blood transfusion & $12(36.4 \%)$ \\
\hline Renal failure & $23(69.7 \%)$ \\
\hline Stroke & $6(18.2 \%)$ \\
\hline Sepsis & $4(12.1 \%)$ \\
\hline 30-day mortality & $6(18.2 \%)$ \\
\hline 180-day mortality & $15(45.5 \%)$ \\
\hline 30-day favorable neurological outcome (CPC 1-2) & $16(48.5 \%)$ \\
\hline 180-day favorable neurological outcome (CPC 1-2) & $17(51.5 \%)$ \\
\hline
\end{tabular}

*Mild therapeutic hypothermia; PCI - percutaneous coronary intervention, CABG - coronary artery bypass grafting, STEMI - ST-elevation myocardial infarction, $T I M I$ - thrombolysis in myocardial infarction, CPC - cerebral performance category. 
was performed using Kaplan-Meier curves followed by the log-rank test. All analyses were performed using IBM SPSS Statistics and Statistica 13.0 software. $P$-values of $<0.05$ were considered statistically significant.

\section{Results}

Baseline characteristics of study participants are shown in Table I. The all-cause mortality among study participants at 30-day and 180-day follow-up was $18.2 \%$ and $45.5 \%$ respectively. Sixteen $(48.5 \%)$ out of the 33 patients had a favorable neurologic outcome at the 30day observation. At the end of the follow-up period, $17(51.5 \%)$ patients had a favorable neurologic outcome. Laboratory findings on admission, during, and after MTH are summarized in Table II.

There were significant differences in median serum levels of RANTES as well as IL-1 $\beta$ measured at T0, T1, and T2: 24.69 (Q1:Q3: 11.8-37.5) ng/ml vs. 3.89 (Q1:Q3: 2.6-7.9) $\mathrm{ng} / \mathrm{ml}$ vs. 2.71 (Q1:Q3: $2.0-5.5) \mathrm{ng} / \mathrm{ml} ; p<0.001$ and 0.196 (Q1:Q3: $0.12-0.38) \mathrm{pg} / \mathrm{ml}$ vs. $0.171(0.07-0.31) \mathrm{pg} / \mathrm{ml}$

Table II. Standard laboratory, coagulation parameters, peripheral blood gas analysis, myocardial injury markers and immune system parameters on admission (T0) during mild therapeutic hypothermia (MTH) (12-24 h; T1) and during rewarming after MTH (48-72 h; T2)

\begin{tabular}{|c|c|c|c|}
\hline Variables & TO & $\mathrm{T} 1$ & $\mathrm{~T} 2$ \\
\hline $\mathrm{WBC}\left[\times 10^{-3} / \mu \mathrm{l}\right]$ & $14.6 \pm 5.9$ & $12.1 \pm 4.4$ & $12.3 \pm 4.5$ \\
\hline $\mathrm{RBC}\left[\times 10^{-6} / \mu \mathrm{l}\right]$ & $4.6 \pm 0.5$ & $4.1 \pm 0.5$ & $3.7 \pm 0.5$ \\
\hline $\mathrm{Hb}[\mathrm{g} / \mathrm{dl}]$ & $13.9 \pm 1.5$ & $12.5 \pm 1.6$ & $11.1 \pm 1.3$ \\
\hline $\operatorname{PLT}\left[\times 10^{-3} / \mu \mathrm{l}\right]$ & $187.8 \pm 49.5$ & $150.4 \pm 42.4$ & $134.2 \pm 46.4$ \\
\hline Creatinine $[\mu \mathrm{mol} / \mathrm{l}]$ & $127.5 \pm 89.3$ & $104.1 \pm 109.1$ & $119.7 \pm 87.6$ \\
\hline GFR & $56.8 \pm 18.0$ & $77.2 \pm 27.4$ & $66.7 \pm 27.7$ \\
\hline BNP [pg/ml] & $2596.1 \pm 7532.5$ & $3690.5 \pm 7009.3$ & $5994.0 \pm 8054.0$ \\
\hline Amylase [U/I] & $99.9 \pm 71.0$ & $232.3 \pm 266.3$ & $158.9 \pm 140.8$ \\
\hline $\operatorname{ALT}[\mathrm{U} / \mathrm{I}]$ & $130.9 \pm 141.1$ & $121.2 \pm 108.4$ & $78.5 \pm 45.4$ \\
\hline $\mathrm{AST}[\mathrm{U} / \mathrm{l}]$ & $139.2 \pm 121.2$ & $227.0 \pm 222.3$ & $124.3 \pm 80.3$ \\
\hline Bilirubin $[\mu \mathrm{mol} / \mathrm{l}]$ & $8.6 \pm 3.7$ & $9.0 \pm 4.8$ & $8.6 \pm 5.6$ \\
\hline ALP [U/l] & $91.7 \pm 42.6$ & $68.3 \pm 36.6$ & $71.5 \pm 28.3$ \\
\hline CRP $[\mathrm{mg} / \mathrm{l}]$ & $3.3 \pm 4.7$ & $27.4 \pm 18.5$ & $165.6 \pm 74.3$ \\
\hline \multicolumn{4}{|c|}{ Coagulation parameters: } \\
\hline INR & $1.2 \pm 0.5$ & $1.2 \pm 0.5$ & $1.2 \pm 0.4$ \\
\hline $\mathrm{DD}[\mu \mathrm{g} / \mathrm{l}]$ & $15453.2 \pm 13702.7$ & $2631.9 \pm 2696.4$ & $1232.5 \pm 1103.1$ \\
\hline Fibrinogen $[\mathrm{g} / \mathrm{l}]$ & $3.0 \pm 0.8$ & $3.1 \pm 0.9$ & $5.0 \pm 1.2$ \\
\hline \multicolumn{4}{|c|}{ Peripheral blood gas analysis: } \\
\hline $\mathrm{pCO}_{2}[\mathrm{~mm} \mathrm{Hg}]$ & $40.7 \pm 7.3$ & $40.3 \pm 7.4$ & $39.6 \pm 8.4$ \\
\hline $\mathrm{pO}_{2}[\mathrm{~mm} \mathrm{Hg}]$ & $223.1 \pm 102.9$ & $145.9 \pm 35.3$ & $116.5 \pm 30$ \\
\hline $\mathrm{pH}$ value & $7.28 \pm 0.10$ & $7.30 \pm 0.08$ & $7.38 \pm 0.07$ \\
\hline Lactate $[\mathrm{mmol} / \mathrm{l}]$ & $5.0 \pm 2.9$ & $2.5 \pm 2.2$ & $1.4 \pm 0.7$ \\
\hline \multicolumn{4}{|c|}{ Myocardial injury markers: } \\
\hline hs-TnT [ng/ml] & $0.217 \pm 0.170$ & $1.992 \pm 2.895$ & $1.524 \pm 2.276$ \\
\hline CK-MB [U/l] & $77.0 \pm 47.2$ & $267.0 \pm 271.2$ & $106.0 \pm 98.1$ \\
\hline CK [U/I] & $381.3 \pm 340.6$ & $3307.8 \pm 3101.2$ & $2717.7 \pm 2133.2$ \\
\hline
\end{tabular}

$A L P$ - alkaline phosphatase, $A L T$ - alanine transaminase, AST - aspartate transaminase, BNP - B-type natriuretic peptide, CK - creatine kinase, CK-MB - creatine kinase-MB (CK-MB), CRP - C reactive protein, DD - D-dimer, GFR - glomerular filtration rate, INR - international normalized ratio, Hb - hemoglobin, hs-TnT high-sensitivity troponin $T, p C \mathrm{O}_{2}$ - partial pressure of carbon dioxide, $\mathrm{PO}_{2}$ - partial pressure of oxygen, $P L T$ - platelets, RBC - red blood cell, WBC-white blood cells. 
vs. 0.214 (Q1:Q3: $0.15-0.41) \mathrm{pg} / \mathrm{ml} ; p=0.034$, respectively (Figures $1 \mathrm{~A}, \mathrm{~B}$ ).

A statistically significant correlation was observed between CCL5/RANTES and IL-1 $\beta$ levels at T0 ( $r=-0.360$; $p=0.045$ ) (Figure $2 \mathrm{~A}$ ), while there was no statistically significant correlation between these cytokines at T1 and T2 $(r=-0.208 ; p=0.252$ and $r=-0.139 ; p=0.473$, respectively).

CCL5/RANTES level correlated significantly with brain natriuretic protein (BNP) value at TO $(r=-0.521 ; p=$ 0.003) (Figure $2 \mathrm{~B}$ ), while an inverse correlation was observed for IL-1 $\beta$ at T1 and BNP level at T1 $(r=0.478 ; p=$ 0.008) (Figure $2 \mathrm{C}$ ). IL-1 $\beta$ also correlated with fibrinogen level at T1 $(r=-0446 ; p=0.012)$ (Figure $2 \mathrm{D})$.

There was a significant correlation between IL-1 $\beta$ and SYNTAX score at T1 $(r=0.378 ; p=0.033)$, whereas no correlation between CCL5/RANTES level and SYNTAX score was observed.

Maximum high-sensitivity troponin and CK-MB levels negatively correlated with CCL5/RANTES level at T2 ( $r=$ $-0.594 ; p<0.001$ and $r=-0.389 ; p=0.030$, respectively), but not with IL-1 $\beta$ (Figures $3 \mathrm{~A}, \mathrm{~B}$ ).

Kaplan-Meier survival curves for medians of CCL5/ RANTES and IL-1 $\beta$ levels at TO and T1 were calculated for 30-day and 180-day mortality.

For 30-day survival curves, the levels of IL-1 $\beta$ at T0 and T1 showed a trend to significance with all-cause mortality rate ( $p=0.078 ; p=0.079$, respectively) (Figure $4 \mathrm{~A} a, b)$. Levels of CCL5/RANTES at T0 and T1 did not have an influence on 30-day mortality $(p=0.284$; $p=0.351$, respectively) (Figure $4 \mathrm{~A} c, \mathrm{~d}$ ).

For 180-day survival curves, the level of IL-1 $\beta$ at T1 significantly associated with all-cause mortality ( $p=$ 0.028 ) (Figure $4 \mathrm{~B}$ a), whereas IL-1 $\beta$ level at T0 as well as CCL5/RANTES levels at T0 and T1 did not show a statisti-

A

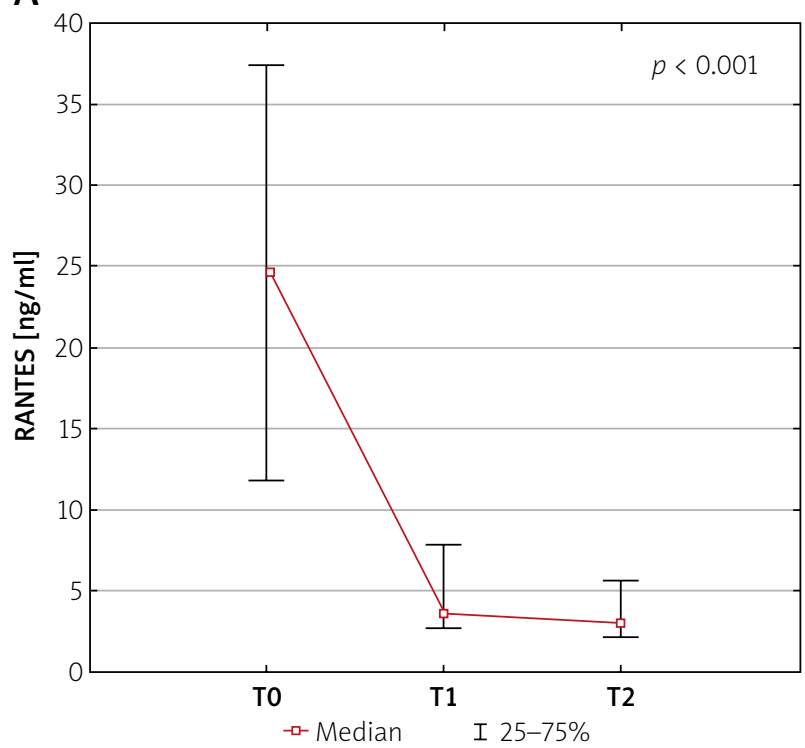

cally significant association with 180-day mortality ( $p=$ 0.391; $p=0.758 ; p=0.502$, respectively) (Figure $4 \mathrm{~B} b-\mathrm{d}$ ).

\section{Discussion}

To our knowledge, this is the first prospective study investigating chemokines which regulate immune cell vascular chemotaxis, including CCL5/RANTES and IL-1 $\beta$, in MTH in patients with cardiac arrest due to ACS treated with $\mathrm{PCl}$.

The main finding of our study is that there are important differences in levels of CCL5/RANTES and IL- $1 \beta$ with regard to time frames. We observed a clear and steady decrease in CCL5/RANTES levels from T0 to T2. This supports the relationship of a chemokine CCL5/RANTES early increase which typically accompanies activation of an intravascular inflammatory response [20].

CCL5/RANTES is mainly produced by T cells, endothelial cells, platelets, and smooth muscle cells and stimulates leukocytes to migrate into inflammatory tissue or into the wall of an injured artery [21]. When released from activated platelets, CCL5/RANTES triggers monocyte migration and arrest to the endothelium in the inflamed or atherosclerotic area [22], and has a significant effect on atherosclerotic lesion size and plaque development. Furthermore, CCL5/RANTES has a role in plaque destabilization and remodeling [23].

In this context, our results suggest that MTH leads to a rapid reduction in CCL5/RANTES levels, which might have a beneficial effect on decreasing the inflammatory response. The same effect was observed for IL-1 $\beta$ during MTH. However, the effect of CCL5/RANTES reduction remained even after patient rewarming, whereas IL-1 $\beta$ levels decreased at T1 and then increased after rewarming of the patient. This might mimic the so-

B

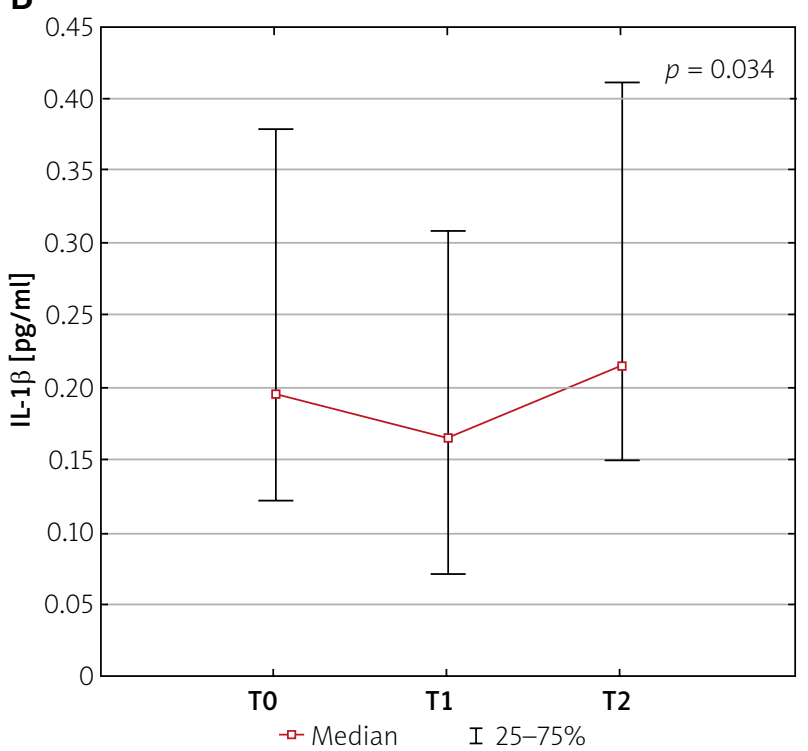

Figure 1. Serum RANTES and IL-1 $\beta$ levels at T0, T1, and T2 
A

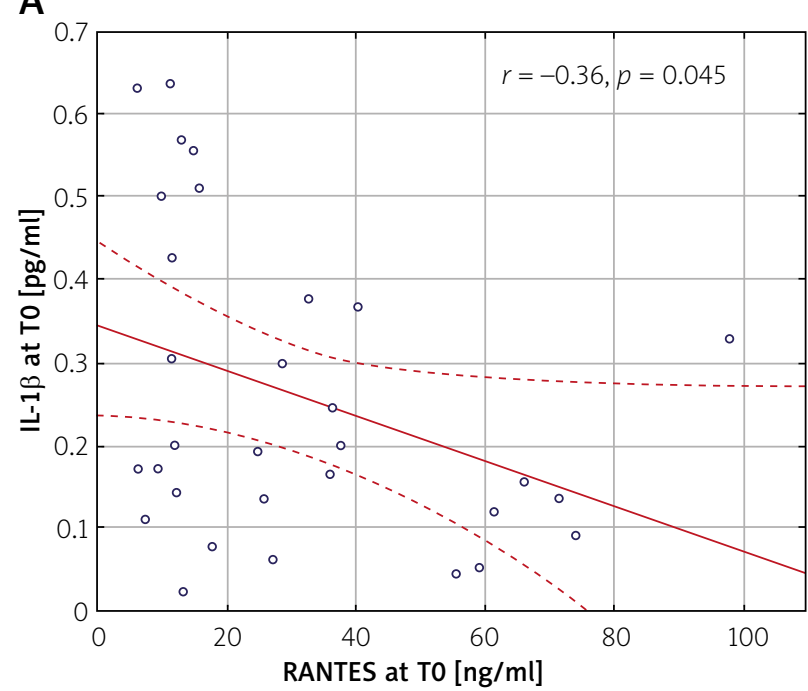

C

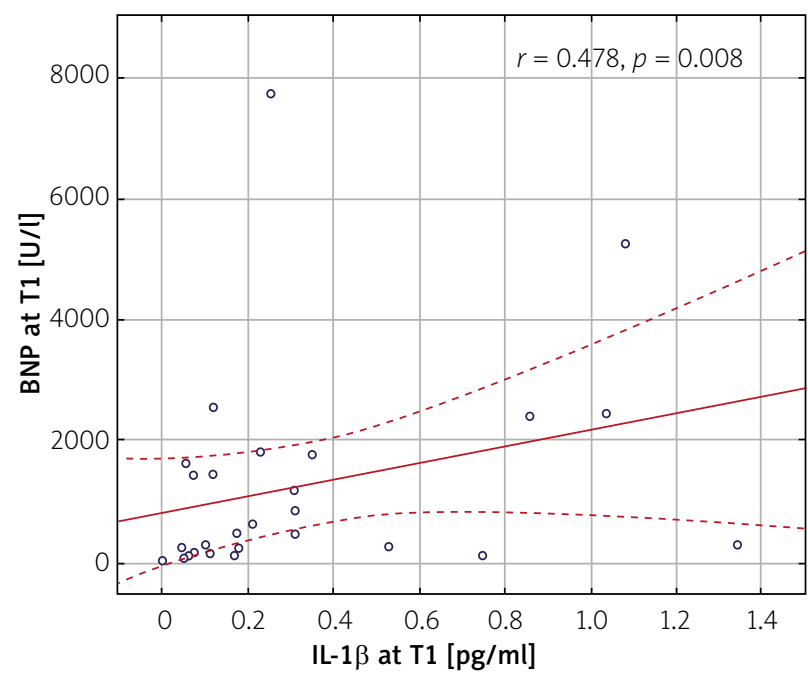

B

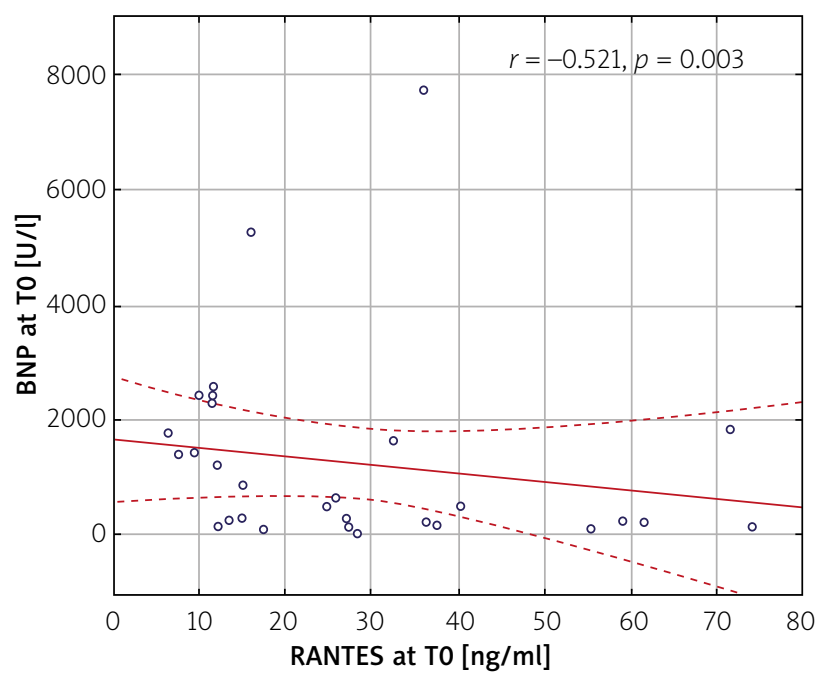

D

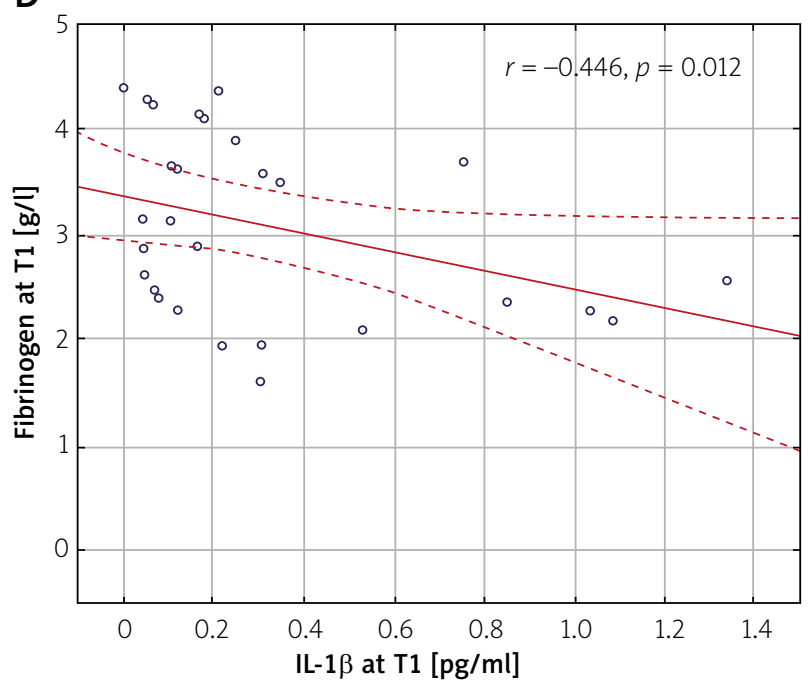

Figure 2. Correlations of RANTES and IL-1 $\beta$ cytokines with regard to laboratory parameters

A

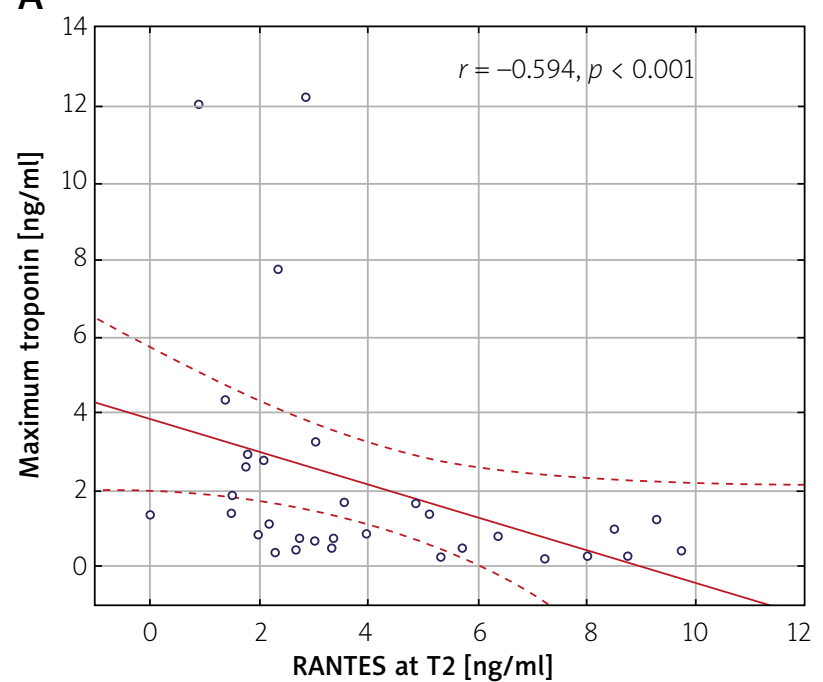

B

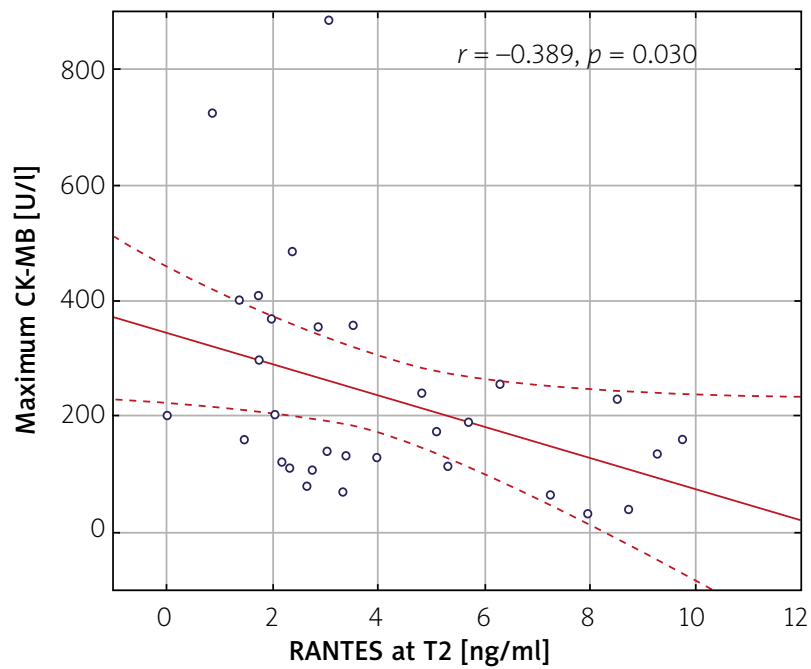

Figure 3. Correlations of RANTES levels at T2 with regard to maximum high-sensitivity troponin and CK-MB levels 
A a

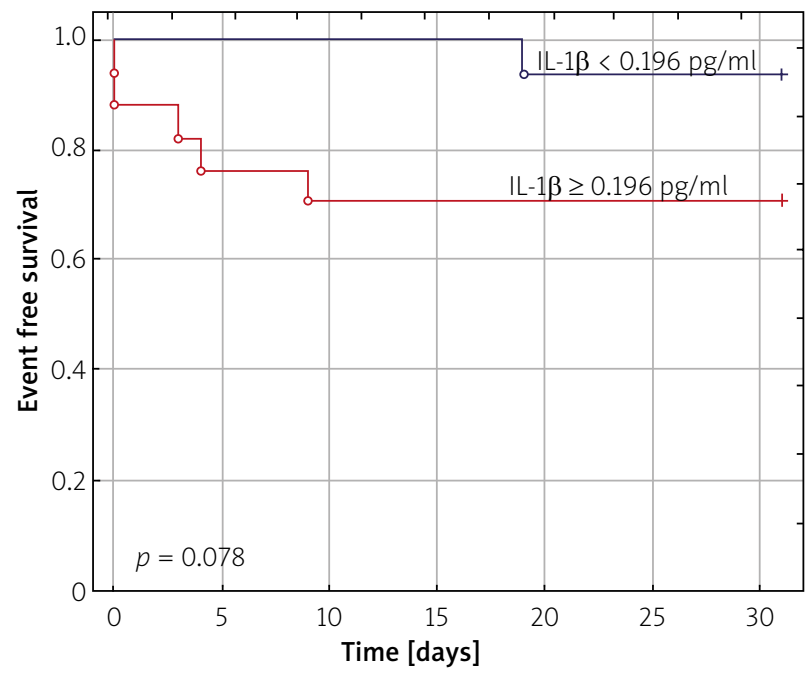

C

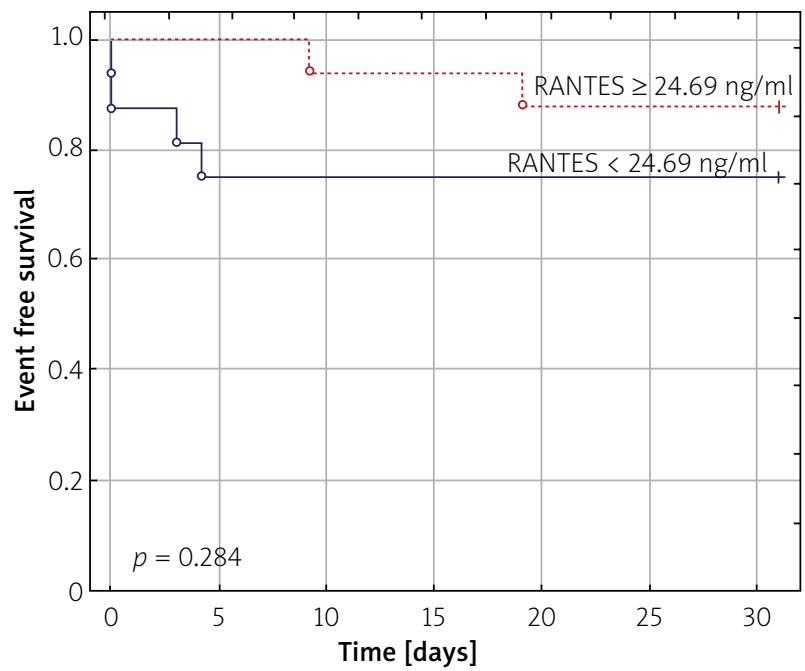

b

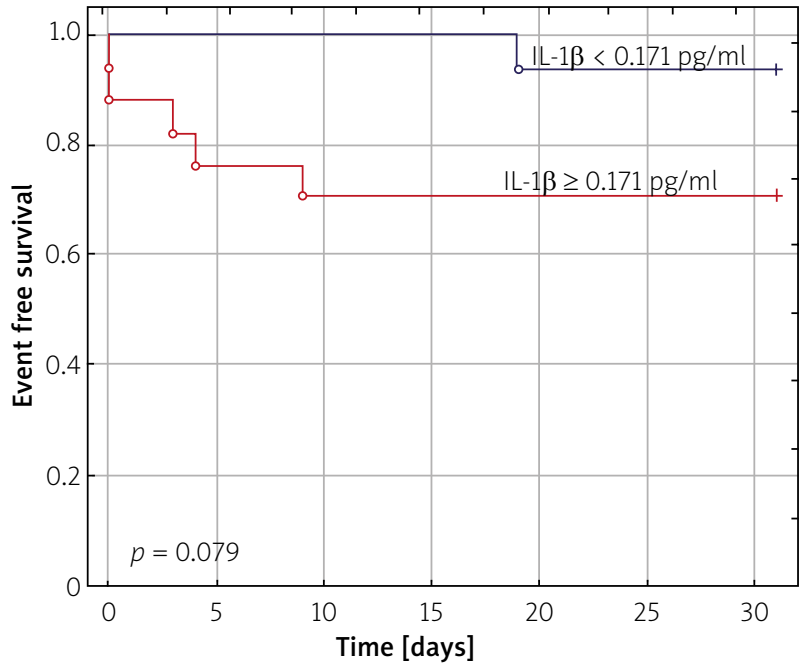

d

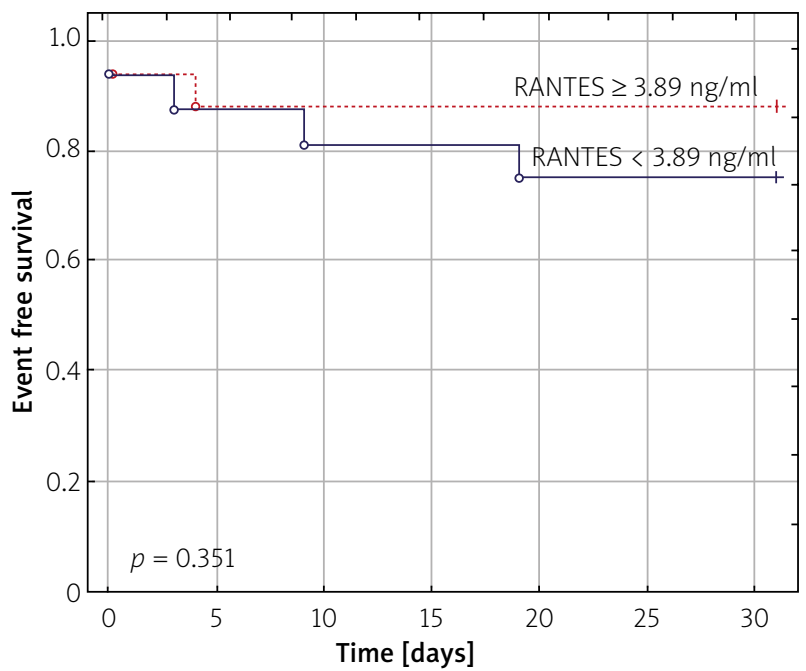

Figure 4. A - Kaplan-Meier curves for 30-day mortality stratified according to median levels of IL-1 13 at TO (a) and T1 (b) and RANTES at TO (c) and T1 (d)

called sepsis-like syndrome, along with an increase in CRP levels, but not in white blood cell count. In line with our findings, MTH was associated with a reduction in immune cell infiltration, apoptosis, IL-1 $\beta$, and IL-6. Hypothermia reduced myocardial damage and dysfunction after cardiopulmonary resuscitation, possibly via a reduced rate of apoptosis and pro-inflammatory cytokine expression [24].

Of note, it was previously shown that other inflammatory markers including cytokine IL- 6 and procalcitonin levels in patients treated with MTH were associated with higher mortality rates measured $24 \mathrm{~h}$ after out-of-hospital SCA $[25,26]$. The CRP levels on admission were associated with poorer outcomes in a similar group of patients [27]. Our data support these findings, as IL-1 $\beta$ levels were associated with 180-day mortality.

Cavusoglu et al. [28] reported that low baseline CCL5/ RANTES levels were an independent predictor of cardiac mortality, with a survival rate of $87.3 \%$ in the lowest tertile of CCL5/RANTES values, compared with $94 \%$ in the upper $1^{\text {st }}$ and $2^{\text {nd }}$ tertile ( $p=0.0298$ by log-rank test) in patients referred for coronary angiography [14, 18, 29].

Lipkova et al. observed decreased serum CCL5/ RANTES levels in ACS patients, which were associated with the severity of myocardial infarction and progression, with the lowest levels seen in cardiogenic shock patients (cutoff level $\geq 80.4 \mathrm{ng} / \mathrm{ml}$ ). This strongly suggests a role for CCL5/RANTES as a potential biomarker of cardiogenic shock and acute heart failure in the post-ACS hospitalization phase [30].

In ACS patients with induced MTH, CCL5/RANTES levels did not correlate with 30-day or 180-day mortality. This might be reflected by the fact that the CCL5/RANTES impact on mortality was diminished by a decreased inflammatory process and prohibition of further cardiac remodeling. Of note, CCL5/RANTES levels correlated with 
B a

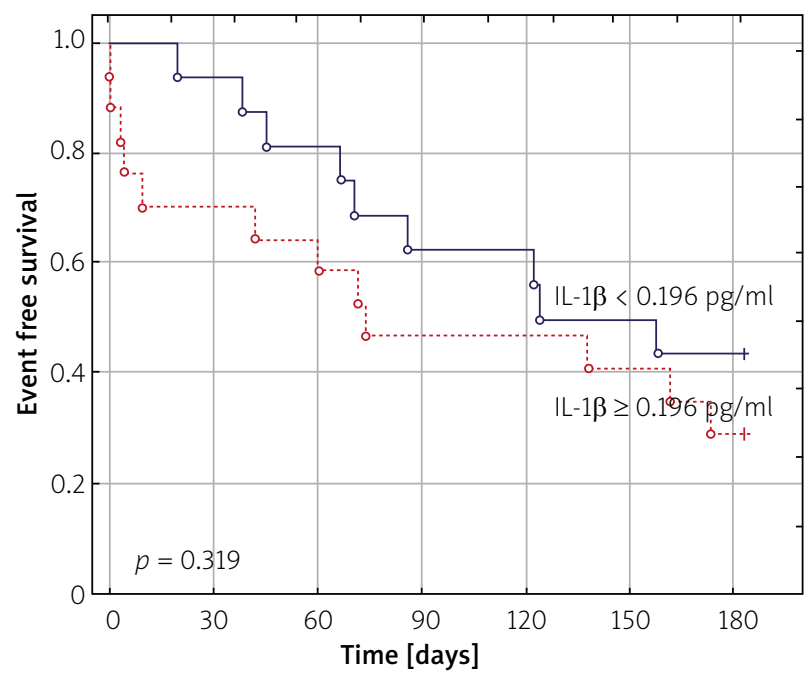

C

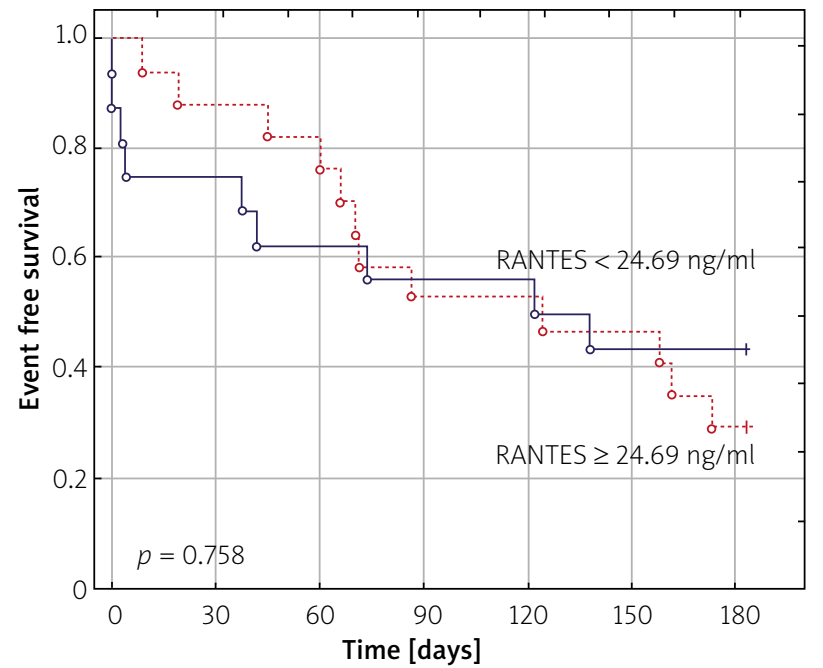

b

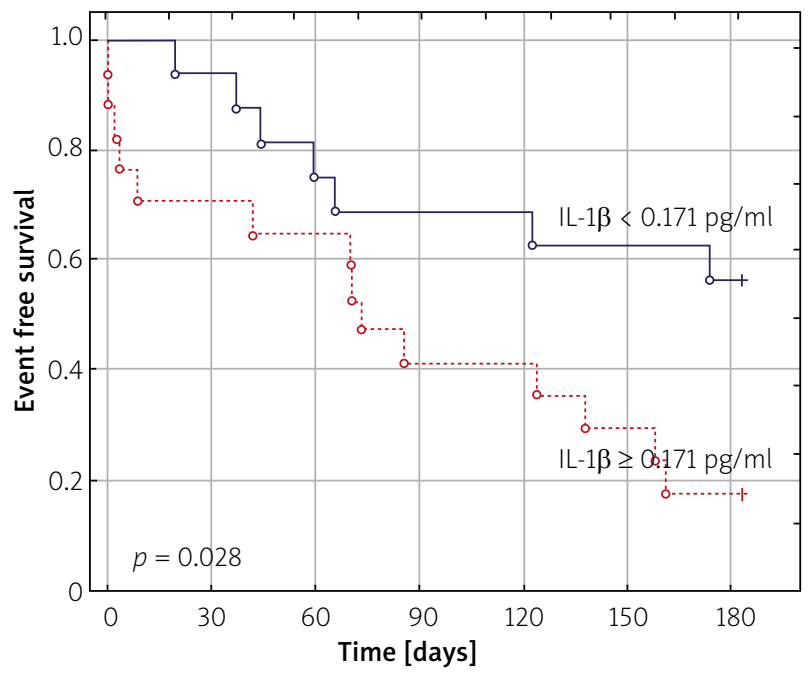

d

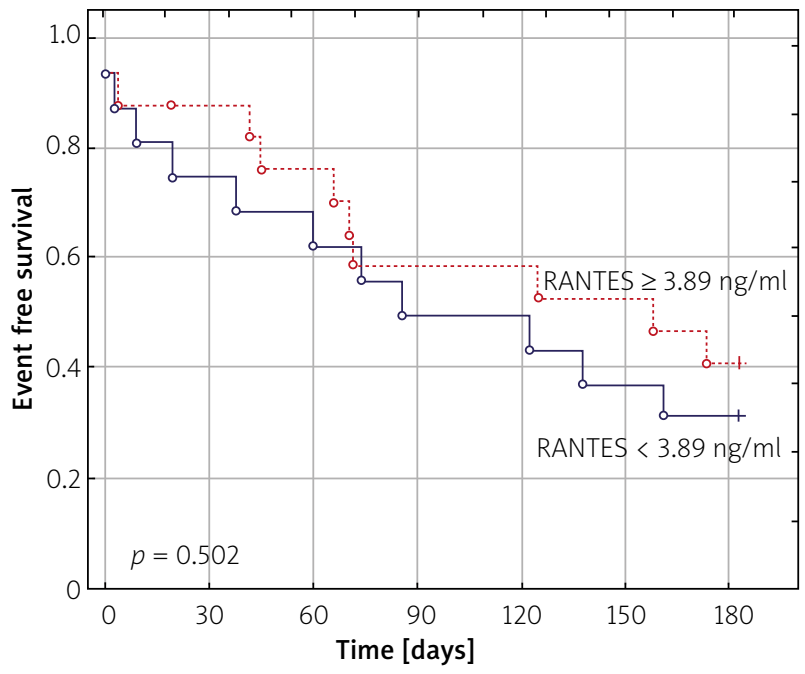

Figure 4. Cont. B - Kaplan-Meier curves for 180-day mortality stratified according to median levels of IL-1 $\beta$ at TO (a) and T1 (b) and RANTES at TO (c) and T1 (d)

cardiac injury and heart failure markers, which still make them potentially valuable, provoking the need for further investigation.

Taken together, this suggests a potentially important role for CCL5/RANTES and IL-1 $\beta$ in patients with ACS and cardiogenic shock. All of the above-mentioned biomarkers correlate with post-MI survival and have an impact on short- and long-term survival [31].

\section{Study limitations}

The study group was relatively small and no control group was used due to ethical reasons and the critical status of all patients. However, the overall group size was sufficient to detect correlations. We assessed cytokine levels only via serum gene expression and not through left ventricular tissue gene expression.

\section{Conclusions}

The chemokine CCL5/RANTES is present in early stages of the inflammatory process in out-of-hospital cardiac arrest patients, then significantly decreases during MTH. The MTH leads to a decrease in the inflammatory response as measured by IL-1 $\beta$. The level of IL-1 $\beta$ measured during MTH was significantly associated with longterm all-cause mortality. CCL5/RANTES levels correlated with myocardial injury and BNP levels.

\section{Acknowledgments}

Support for this article was provided by Jagiellonian University Collegium Medicum Grant K/ZDS/004598.

\section{Conflict of interest}

The authors declare no conflict of interest. 


\section{References}

1. Strzelecki Z, Szymborski J. Zachorowalność i umieralność na choroby krążenia a sytuacja demograficzna Polski. Czech-Matuszewska W. Rządowa Rada Ludnościowa, Warszawa 2015.

2. Gräsner JT, Lefering R, Koster RW, et al. EuReCa ONE Collaborators. EuReCa ONE-27 Nations, ONE Europe, ONE Registry: a prospective one month analysis of out-of-hospital cardiac arrest outcomes in 27 countries in Europe. Resuscitation 2016; 105: 188-95.

3. Mędrzycka-Dąbrowska WA, Czyż-Szybenbej K, Kwiecień-Jaguś K, et al. Prediction of cognitive dysfunction after resuscitation a systematic review. Adv Interv Cardiol 2018; 14: 225-32.

4. Neumar RW, Nolan JP, Adrie C, et al. Post-cardiac arrest syndrome. Circulation 2008; 118: 2452-83.

5. Hypothermia after Cardiac Arrest Study Group (HACA). Mild therapeutic hypothermia to improve the neurologic outcome after cardiac arrest. N Engl J Med 2002; 346: 549-56.

6. Bernard SA, Gray TW, Buist MD, et al. Treatment of comatose survivors of out-of-hospital cardiac arrest with induced hypothermia. N Engl J Med 2002; 346: 557-63.

7. Saigal S, Sharma JP, Dhurwe R, et al. Targeted temperature management: current evidence and practices in critical care. Indian J Crit Care Med 2015; 19: 537-46.

8. Serpytis P, Smigelskaite A, Kibarskis A, et al. Successful treatment of a young woman with acute complicated myocardial infarction. Adv Interv Cardiol 2013; 9: 369-75.

9. Nolana JP, Soar J, Cariou A, et al. European Resuscitation Council and European Society of Intensive Care Medicine Guidelines for Post-resuscitation Care 2015. Section 5 of the European Resuscitation Council Guidelines for Resuscitation 2015. Resuscitation 2015; 95: 202-22.

10. Adrie C, Adib-Conquy M, Laurent I, et al. Successful cardiopul-monary resuscitation after cardiac arrest as "sepsis-like" syndrome. Circulation 2002; 106: 562-8.

11. de Jager SCA, Bongaerts BWC, Weber M, et al. Chemokines CCL3/MIP1, CCL5/RANTES and CCL18/PARC are independent risk predictors of short-term mortality in patients with acute coronary syndromes. PLoS One 2012; 7: e45804.

12. Kraaijeveld AO, de Jager SCA, de Jager WJ, et al. CC chemokine ligand-5 (CCL5/RANTES) and CC chemokine ligand-18 (CCL18/ PARC) are specific markers of refractory unstable angina pectoris and are transiently raised during severe ischemic symptoms. Circulation 2007; 116: 1931-41.

13. Podolec J, Niewiara L, Skiba D, et al. Higher levels of circulating naïve CD8+CD45RA+ cells are associated with lower extent of coronary atherosclerosis and vascular dysfunction. Int I Cardiol 2018; 259: 26-30.

14. Podolec J, Kopec G, Niewiara L, et al. Chemokine RANTES is increased at early stages of coronary artery disease. J Physiol Pharmacol 2016; 67: 321-8.

15. Aslan AN, Özcan AN, Ayhan H, et al. Evaluation of local carotid stiffness and inflammatory biomarkers in stable angina pectoris. Adv Interv Cardiol 2017; 13: 122-9.

16. Ibanez B, James S, Agewall S, et al. ESC Scientific Document Group. 2017 ESC Guidelines for the management of acute myocardial infarction in patients presenting with ST-segment elevation: The Task Force for the management of acute myocardial infarction in patients presenting with ST-segment elevation of the European Society of Cardiology (ESC). Eur Heart J 2018; 39: $119-77$.
17. Neumann FJ, Sousa-Uva M, Ahlsson A, et al. ESC Scientific Document Group. 2018 ESC/EACTS Guidelines on myocardial revascularization. Eur Heart J 2019; 40: 87-165.

18. Jennett $B$, Bond $M$. Assessment of outcome after severe brain damage. Lancet 1975; 1: 480-4.

19. Cummins RO, Chamberlain DA, Abramson NS, et al. Recommended guidelines for uniform reporting of data from outof-hospital cardiac arrest: the Utstein Style. A statement for health professionals from a task force of the American Heart Association, the European Resuscitation Council, the Heart and Stroke Foundation of Canada, and the Australian Resuscitation Council. Circulation 1991; 84: 960-75.

20. Conti P, DiGioacchino M. MCP-1 and RANTES are mediators of acute and chronic inflammation. Allergy Asthma Proc 2001; 22: 133-7.

21. Appay V, Rowland-Jones SL. RANTES: a versatile and controversial chemokine. Trends Immunol 2001; 22: 83-7. 9.

22. von Hundelshausen P, Weber KS, Huo Y, et al. RANTES deposition by platelets triggers monocyte arrest on inflamed and atherosclerotic endothelium. Circulation 2001; 103: 1772-7.

23. Aukrust P, Yndestad A, Smith C, et al. Chemokines in cardiovascular risk prediction. Thromb Haemost 2007; 97: 748-54.

24. Meybohm P, Gruenewald M, Albrech M, et al. Hypothermia and postconditioning after cardiopulmonary resuscitation reduce cardiac dysfunction by modulating inflammation, apoptosis and remodeling. Plos One 2009; 4: e7588.

25. Bro-Jeppesen J, Kjaergaard J, Wanscher M, et al. Systemic inflammatory response and potential prognostic implications after out-of-hospital cardiac arrest: a substudy of the target temperature management trial. Crit Care Med 2015; 43: 1223-32.

26. Bro-Jeppesen J, Kjaergaard J, Stammet P, et al.; TTM-Trial Investigators. Predictive value of interleukin-6 in post-cardiac arrest patients treated with targeted temperature management at $33^{\circ} \mathrm{C}$ or $36^{\circ} \mathrm{C}$. Resuscitation 2016; 98: 1-8.

27. Dell'anna AM, Bini Viotti J, Beumier M, et al. C-reactive protein levels after cardiac arrest in patients treated with therapeutic hypothermia. Resuscitation 2014; 85: 932-8.

28. Cavusoglu E, Eng C, Chopra V, et al. Low plasma RANTES levels are an independent predictor of cardiac mortality in patients referred for coronary angiography. Arterioscler Thromb Vasc Biol 2007; 27: 929-35.

29. Podolec J, Baran J, Siedlinski M, et al. Serum rantes, transforming growth factor-beta1 and interleukin-6 levels correlate with cardiac muscle fibrosis in patients with aortic valve stenosis. J Physiol Pharmacol 2018; 69. doi: 10.26402/jpp.2018.4.12.

30. Lipkova J, Parenica J, Duris K, et al. Association of circulating levels of RANTES and -403G/A promoter polymorphism to acute heart failure after STEMI and to cardiogenic shock. Clin Exp Med 2015; 15: 405-14.

31. Bertinchant JP, Ledermann B, Schmutz L, et al. Diagnostic and prognostic significance of CK-MB, troponins, CRP, BNP and/or NT-proBNP in coronary angioplasty. Elevation mechanisms and clinical implications. Arch Mal Coeur Vaiss 2007; 100: 925-33. 